\title{
Breast tumor copy number aberration phenotypes and genomic instability
}

Jane Fridlyand ${ }^{\dagger 1,2}$, Antoine M Snijders ${ }^{\dagger 2,3}$, Bauke Ylstra ${ }^{\dagger 3,4}, \mathrm{Hua} \mathrm{Li}^{2,3}$, Adam Olshen ${ }^{2,5}$, Richard Segraves ${ }^{6}$, Shanaz Dairkee ${ }^{2,7}$, Taku Tokuyasu ${ }^{2,3}$, Britt Marie Ljung2,8, Ajay N Jain2,3, Jane McLennan², John Ziegler², Koei Chin 2,6, Sandy Devries²,6, Heidi Feiler ${ }^{2,9}$, Joe W Gray ${ }^{2,9}$, Frederic Waldman 2,6, Daniel Pinkel 2,6 and Donna G Albertson*2,3,6

Address: ${ }^{1}$ Department of Epidemiology and Biostatistics, University of California San Francisco, San Francisco, San Francisco, CA 94143, USA, ${ }^{2}$ University of California San Francisco Comprehensive Cancer Center, San Francisco, CA 94143, USA, ${ }^{3}$ Cancer Research Institute, University of California San Francisco, San Francisco, CA 94143-0808, USA, ${ }^{4}$ Micro Array Core Facility, VUMC University Medical Center, 1081BT Amsterdam, The Netherlands, ${ }^{5}$ Department of Epidemiology and Biostatistics, Memorial Sloan-Kettering Cancer Center, New York, New York 20021, USA, ${ }^{6}$ Department of Laboratory Medicine, University of California San Francisco, San Francisco, CA 94143-0808, USA, ${ }^{7}$ Geraldine Brush Cancer Research Institute, California Pacific Medical Center, San Francisco, California 94115, USA, ${ }^{8}$ Department of Pathology, University of California

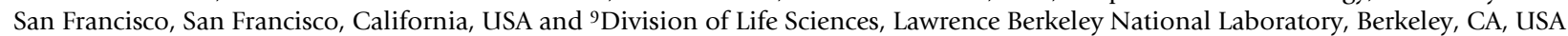

Email: Jane Fridlyand - fridlyand@cc.ucsf.edu; Antoine M Snijders - snijders@cc.ucsf.edu; Bauke Ylstra - b.ylstra@vumc.nl;

Hua Li - hli@cc.ucsf.edu; Adam Olshen - olshena@mskcc.org; Richard Segraves - segraves@cc.ucsf.edu;

Shanaz Dairkee - shanaz@cooper.cpmc.org; Taku Tokuyasu - tokuyasu@cc.ucsf.edu; Britt Marie Ljung - britt-marie.ljung@ucsf.edu;

Ajay N Jain - ajain@cc.ucsf.edu; Jane McLennan - jmclennan2@cc.ucsf.edu; John Ziegler - jziegler@cc.ucsf.edu; Koei Chin - kchin@cc.ucsf.edu; Sandy Devries - devries@cc.ucsf.edu; Heidi Feiler - HSFeiler@lbl.gov; Joe W Gray - jwgray@lbl.gov; Frederic Waldman - waldman@cc.ucsf.edu; Daniel Pinkel - Pinkel@cc.ucsf.edu; Donna G Albertson* - albertson@cc.ucsf.edu

* Corresponding author †Equal contributors

Published: 18 April 2006

BMC Cancer2006, 6:96 doi:10.1 186/147|-2407-6-96
Received: 15 February 2006

Accepted: 18 April 2006

This article is available from: http://www.biomedcentral.com/I47I-2407/6/96

(C) 2006Fridlyand et al; licensee BioMed Central Ltd.

This is an Open Access article distributed under the terms of the Creative Commons Attribution License (http://creativecommons.org/licenses/by/2.0), which permits unrestricted use, distribution, and reproduction in any medium, provided the original work is properly cited.

\begin{abstract}
Background: Genomic DNA copy number aberrations are frequent in solid tumors, although the underlying causes of chromosomal instability in tumors remain obscure. Genes likely to have genomic instability phenotypes when mutated (e.g. those involved in mitosis, replication, repair, and telomeres) are rarely mutated in chromosomally unstable sporadic tumors, even though such mutations are associated with some heritable cancer prone syndromes.
\end{abstract}

Methods: We applied array comparative genomic hybridization (CGH) to the analysis of breast tumors. The variation in the levels of genomic instability amongst tumors prompted us to investigate whether alterations in processes/genes involved in maintenance and/or manipulation of the genome were associated with particular types of genomic instability.

Results: We discriminated three breast tumor subtypes based on genomic DNA copy number alterations. The subtypes varied with respect to level of genomic instability. We find that shorter telomeres and altered telomere related gene expression are associated with amplification, implicating telomere attrition as a promoter of this type of aberration in breast cancer. On the other hand, the numbers of chromosomal alterations, particularly low level changes, are associated with altered expression of genes in other functional classes (mitosis, cell cycle, DNA replication and repair). Further, although loss of function instability phenotypes have been demonstrated for 
many of the genes in model systems, we observed enhanced expression of most genes in tumors, indicating that over expression, rather than deficiency underlies instability.

Conclusion: Many of the genes associated with higher frequency of copy number aberrations are direct targets of E2F, supporting the hypothesis that deregulation of the Rb pathway is a major contributor to chromosomal instability in breast tumors. These observations are consistent with failure to find mutations in sporadic tumors in genes that have roles in maintenance or manipulation of the genome.

\section{Background}

Genomic DNA copy number aberrations are frequent in solid tumors [1]. The wide range in the number and types of chromosome level alterations are likely to reflect the different solutions taken by individual tumors to escape normal protective mechanisms. Thus, the spectrum of alterations is likely to reflect a composite of selection and particular failures in genome surveillance mechanism(s). The interplay between selection and genetic instability in shaping tumor genomes is currently most clearly established in tumors with defects in mismatch repair. These tumors have a high frequency of nucleotide sequence level aberrations, fewer DNA copy number alterations and characteristic histological phenotype [1]. On the other hand, less is known about specific gene defects that give rise to chromosome level aberrations in tumors. Mutations in genes encoding proteins involved in mitosis and DNA damage sensing and repair mechanisms, which are associated with chromosomal level instability have been identified in cancer-prone syndromes, including $A T M$, TP53, BRCA1, BRCA2, NBS1 and BUB1B, however they are rarely mutated in sporadic tumors [2,3]. Similarly, searches for mutations in genes that participate in maintenance or manipulation of the genome (e.g. genes involved in DNA repair, replication, spindle checkpoints etc.) have found only a small number of mutations in tumors [3]. Nevertheless, deregulation of functions that maintain genome stability appears to occur early in tumors, as activation of the DNA damage checkpoint, possibly in response to DNA replication stress, is evident in premalignant lesions $[4,5]$. Similarly, telomere shortening is observed in pre-malignant lesions, supporting a role for telomere dysfunction early in tumor development [6]. Other proposed routes to instability include deregulation of CCNE1 and AURKA expression through loss of function of FBXW7 (hCdc4) [7] and more global alteration in gene expression due to deregulation of the $\mathrm{Rb}$ pathway [8]. The foregoing discussion suggests that failures in a number of different processes that maintain genome integrity could contribute to the wide variety of genomic alterations in solid tumors. Often these aberrations include net gain or loss of whole chromosomes (aneuploidy) or parts of chromosomes. Gene amplification, defined as a copy number increase of a restricted region of a chromosome arm may also occur. Here we investigated the numbers and types of copy number alterations in tumors and whether they were associated with differential expression of genes likely to play a role in manipulation or maintenance of the genome. These studies found three subtypes of breast tumors distinguished by copy number aberrations. Telomere dysfunction was implicated in the propensity to amplify, since shorter telomeres and differential expression of genes involved in telomere maintenance were associated with the numbers of amplicons and the presence of at least one amplicon, respectively. On the other hand, the number of lower magnitude gains and losses of chromosomal segments was associated with differential expression of genes involved in processes maintaining or manipulating the genome. These genes are significantly enriched for the known targets of E2F. Furthermore, we observed enhanced expression of most E2F target genes, indicating that over expression rather than deficiency was associated with genetic instability. These observations support the hypothesis that deregulation of the $\mathrm{Rb} / \mathrm{E} 2 \mathrm{~F}$ pathway is a major contributor to chromosomal instability in breast tumors.

\section{Methods \\ Specimens}

Frozen tumor tissue was obtained from the University of California San Francisco Comprehensive Cancer Center Breast Oncology Program Tissue Bank. All specimens were collected under approved protocols from UCSF with patient consent. Patient characteristics are provided in Supplementary Table 1 (Additional file 1). Expression and copy number data from a second set of ductal invasive breast tumors were used and patient characteristics are given in Chin et al. (submitted). The patient groups in both sets were similar in terms of their genomic and pathological characterization.

\section{Extraction of nucleic acids}

Nucleic acids were extracted from tumor blocks as described previously $[9,10]$. Briefly, blocks were trimmed with a razor blade to remove normal tissue and cryosections were obtained from either side of the block to ascertain that tumor cells comprised greater than $70 \%$ of the specimen. DNA was extracted using the QUIamp tissue kits (29304, Qiagen). 
Table I: Recurrent amplicons in breast tumors with examples of some candidate oncogenes

\begin{tabular}{|c|c|c|c|c|c|c|c|}
\hline Tumors & Chr. & Proximal flanking clone & Start (bp) & Distal flanking clone & End (bp) & $\begin{array}{l}\text { Size } \\
\text { (Mb) }\end{array}$ & Candidate Genes \\
\hline S0257; SI 508 & 1 & RPII-235B24 & 50120611 & CTD-2010C4 & 64641284 & 14.5 & JUN \\
\hline $\begin{array}{l}\text { S002I; S0065; SOI27; SOI32; SI534; } \\
\text { SI539 }\end{array}$ & 8 & RPII-2IOFI5 & 36452678 & RPII-262123 & 39744917 & 3.3 & BAGI; FGFRI; TACCI \\
\hline SOOI3; S0257; S1598 & 8 & RPII-|28G 18 & 127638988 & RPII-227F7 & 131013284 & 3.4 & MYC; WISPI \\
\hline SOI32; S0394; SI 524 & II & CTD-2115CI7 & 32421376 & RPII-I8B9 & 40252688 & 7.8 & TRAF6 \\
\hline $\begin{array}{l}\text { S0050; S0065; S008I; SOI 32; S0252; } \\
\text { S0303; SI 534; SI 539; SI } 598\end{array}$ & 11 & CTD-2080119 & 68483419 & RPII-98G24 & 76964746 & 8.5 & CCNDI; FGF4; EMSI; PAKI \\
\hline SOI84; SI 508 & 12 & 12pter & 0 & RPII-272L6 & 5221281 & 5.2 & CCND2; FGF6; DYRK4 \\
\hline S005I; S0052; SOI22; SI 522 & 12 & RPII-I8B8 & 63882712 & RPII-92P22 & 74052886 & 10.2 & $\begin{array}{l}\text { MDM2; DYRK2; YEATS4; } \\
\text { HELB; }\end{array}$ \\
\hline $\begin{array}{l}\text { SO02I; SO043; SO052; S0059; S0257; } \\
\text { S0394; SI 5II; SI 522; SI 526; SI 539 }\end{array}$ & 17 & RPII-58O8 & $34 \mid 47697$ & RPII-87N6 & 38680670 & 4.5 & $\begin{array}{l}\text { STARD3; ERBB2; GRB7; } \\
\text { TOP2A; MMP28 }\end{array}$ \\
\hline S0043; SOI04; S0394 & 17 & $\mathrm{RPII}-110 \mathrm{H} 20$ & 47546526 & RPII-48IC4 & 49705574 & 2.2 & IMP-I; ITGA3 \\
\hline $\begin{array}{l}\text { S000I; S002I; S0043; SOI04; SI 508; } \\
\text { SI5II }\end{array}$ & 17 & RP5-1073FI5 & 58499308 & СТВ-244K7 & 64939230 & 6.4 & BRIPI; GHI; GH2; MAP3K3 \\
\hline SOI27; S0257; S0269 & 18 & RPII-7E5 & $108|7| 40$ & RPII-I0G8 & 17272427 & 6.5 & PTPN2 \\
\hline $\begin{array}{l}\text { SO02I; SO043; SO050; SO05I; S0055; } \\
\text { S0059; SOI22; SI522; SI 545; SI598 }\end{array}$ & 20 & RPII-169A6 & 44424925 & RMC20PI79 & 62702798 & 18.3 & CYP24; ZNF2 I 7; STK6 \\
\hline
\end{tabular}

\section{TP53 sequencing}

Exons 5-8 of TP53 were amplified from genomic DNA and cycle sequencing was carried out as described previously [11].

\section{Array CGH and data processing}

Array CGH, imaging and data analysis were carried out as described previously using arrays of 2464 genomic clones (BAC or P1) each printed in triplicate (HumArray1.14 and HumArray2.0) $[11,12]$. Data processing is described in detail in the Supplementary Methods (Additional file 2) and the array data are available in Supplementary Table 2 (Additional file 3).

\section{Telomere length assessment}

The mean TRF length was measured using the TeloTAGGG telomere length assay kit (Roche Applied Science). Briefly, $1 \mu \mathrm{g}$ genomic DNA was digested with Hinf I and Rsa I restriction enzymes and electrophoretically resolved on $0.8 \%$ agarose/ $1 \mathrm{X}$ TAE. The gels were blotted to a nylon membrane (Positive charged, Roche) and fixed by UVcrosslinking. After hybridization with digoxigenin labeled telomere specific probe, the signals were visualized with an alkaline phosphatase - CDP-Star chemiluminescent system. The filters were exposed to X-ray film and the mean TRF length was calculated using Quantity One software.

\section{Statistical methods}

A detailed description of the methods used for all aspects of the data analysis is provided in the Supplementary Methods (Additional file 2).

\section{Results}

Genomic analysis of breast tumors

Application of array CGH to the analysis of copy number aberrations in 62 sporadic ductal invasive breast tumors and five BRCA1 mutant tumors revealed a number of frequent low level gains and losses (Supplementary Tables 1 and 2, Additional files 1 and 3) and 12 regions of recurrent amplification (Table 1 ). We observed significant differences in the spectrum of aberrations with respect to estrogen receptor (ER) status (Figure 1) consistent with other published reports [13]. We also found that tumors with mutations in exons 5-8 of TP53 showed a higher frequency of alterations, as well as significant differences in the frequency of alteration of certain regions of the genome compared to tumors without mutations as indicated by the t-statistic for each clone (Figure 2). Moreover, we found a highly significant concordance between the test statistic for association of the particular chromosomal regions with TP53 mutation status in our data and an independent set of more than 100 primary breast tumors (Fedele et al., personal communication) (Pearson correlation of 0.53 corresponding to the p-value $<<0.001$ ).

Hierarchical clustering of tumors according to their genome-wide DNA copy number profiles revealed three main branches. Tumors within each of the branches also differed in the number of copy number changes that were present, as well as the frequency of particular aberrations (Figures 3 and 4). The same three clusters were observed in an independent set of breast tumors (Chin et al., submitted), thus confirming our initial observation. The groups in both sets agreed in terms of their genomic and pathological characterization.

At the low end of chromosomal level instability are ER positive tumors ( $\mathrm{n}=7$ tumors, Figure 1A, left branch), 


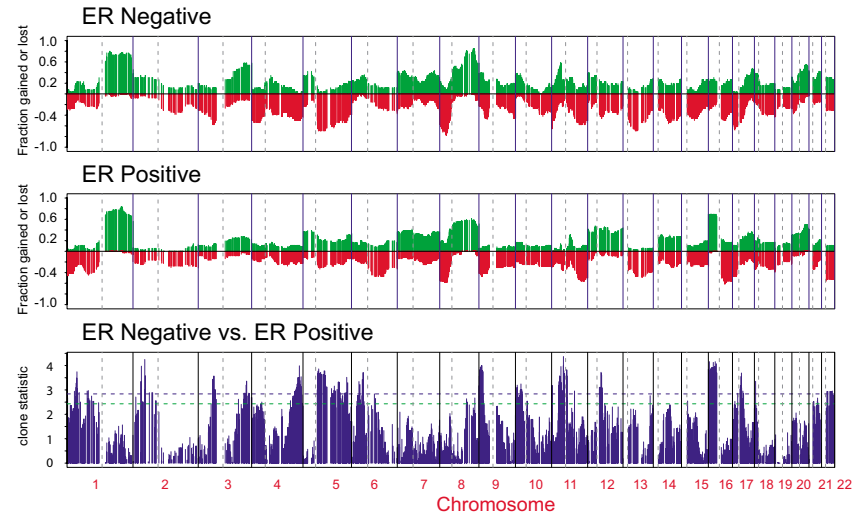

Figure I

Frequency plot of copy number alterations in ER positive and negative tumors. The top two panels show the frequency of gains, indicated by the green bars ranging from 0 to $\mathrm{I}$, and losses, indicated by the red bars ranging from 0 to $\mathrm{I}$, in 62 sporadic breast tumors for each clone. The bottom panel displays the magnitude of the t-statistic for each clone computed based on the smoothed data as described in the Methods. The horizontal dotted lines indicate the statistic cut-off corresponding to the FDR-adjusted p-value of 0.05 (blue) and 0.1 (green).

designated $1 \mathrm{q} / 16 \mathrm{q}$, as their genomes showed very few copy number changes other than gain of $1 \mathrm{q}$ and loss of $16 \mathrm{q}$ (Figure 3A, B, G and 3H, Figure 4). Tumors in this group were exclusively of moderately or well differentiated grade, stage II, and did not recur. These tumors had very high within group similarity with average pairwise Pearson correlation of 0.76 .

At the other extreme of genome instability are sporadic tumors ( $n=16$, Figure $3 A$, middle branch) in which the mean fraction of the genome at altered copy number is greater than 0.6 due to the presence of many low level copy number aberrations (Figure 3A, C, G and 3H). Copy number losses involving chromosomes $3 \mathrm{p}, 4,5 \mathrm{q}, 11 \mathrm{p}$, $14 \mathrm{q}, 15 \mathrm{q}, 17 \mathrm{q}$ and $18 \mathrm{q}$ were more prevalent in this subtype than in others (Figure 4). All but one tumor in this group are ER negative, all were of high grade and patients experienced significantly worse outcome as compared to other groups (Figure 3I). Four had mutations in exons 58 of TP53, accompanied by a copy number loss encompassing the locus (Table 2). In addition this cluster contains all of the familial BRCA1 mutant tumors (Figure 3E and $3 \mathrm{~F}$ ) in our dataset. Similar to BRCA1 mutant tumors, they show a relatively high degree of within group similarity in regions of aberration in spite of the presence of many alterations (see Methods for discussion of statistical analysis, Additional file 2). We refer to this group as "complex" in recognition of their many low level copy number alterations.
The third group ( $n=39$, Figure $3 A$, right branch) comprised of both ER positive and negative tumors is characterized by the presence of low level gains and losses and recurrent amplifications (Figure 3A, D, G and 3H). Gains involving chromosome $8 \mathrm{q}$ and $12 \mathrm{p}$ were more frequent in this group than the others (Figure 4). We refer to this group as "mixed amplifiers." The more frequently occurring amplifications in this group, which occurred predominantly in the ER positive tumors involved $8 \mathrm{p}$, including FGFR1 ( 6 ER positive tumors/ 6 tumors with $8 \mathrm{p}$ amplification), 11q13, including CCND1 ( 8 ER positive tumors/9 tumors with $11 \mathrm{q} 13$ amplification) and regions of $20 \mathrm{q}$ including ZNF217 ( 6 ER positive tumors/9 tumors with $20 \mathrm{q}$ amplification). Within this subtype, amplification of $17 \mathrm{q}$ (ERBB2) was present in both ER positive $(\mathrm{n}=5)$ and ER negative tumors $(n=2)$ (Figure $5 \mathrm{~A}$ and Table 1 ).

\section{Association of copy number aberration types with alterations in processes/genes involved in maintenance and manipulation of the genome}

The discrimination of breast tumor subtypes based on copy number aberrations led us to investigate possible associations of copy number aberration types with alterations in processes/genes involved in maintenance of genome stability. Over expression or depletion of such genes in vitro results in a variety of genome instability phenotypes, including disruptions of chromosome integrity, aberrant mitoses, aberrant cell division, etc. As telomere dysfunction has been widely proposed as a source of genetic instability in tumors, we first investigated the possible association of telomere attrition with copy number aberrations. We determined average telomere length in 28 breast tumors using Southern blotting. We found an inverse correlation between telomere length and number of chromosome arms with amplification (Figure 6, Spearman correlation $=-0.42, \mathrm{p}=0.02)$. Moreover we observed an inverse association between telomere length and the presence of at least one amplicon (median length in amplified samples of 6.3 compared to unamplified, 7.4 ), but the comparison was underpowered and statistically not significant (Wilcoxon rank sum test, $\mathrm{p}=0.25$ ). These observations suggest a role for telomere attrition in promoting amplification in breast tumors.

Next, we investigated whether expression levels of genes that play a role in maintenance or manipulation of the genome varied among tumors with greater or lesser numbers of copy number aberrations. To carry out this analysis we used a second independent set of 101 ductal invasive breast tumors for which copy number profiles and Affymetrix High Throughput Array (HTA) GeneChip ${ }^{\circledR}$ expression data were available (Chin et al., submitted). We determined the number and type of copy number changes in each tumor by counting three types of copy number alterations; copy number changes involving 


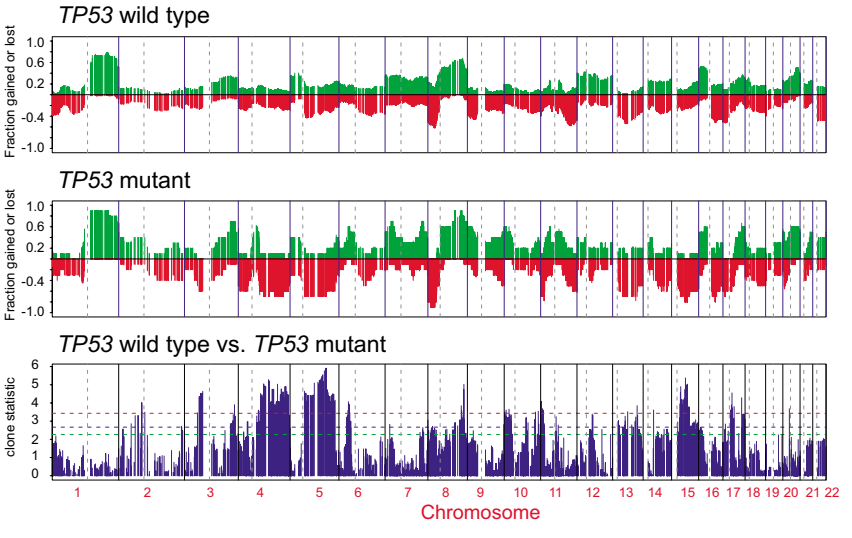

Figure 2

Analysis of TP53 mutation in breast tumors. Frequency plot of copy number changes in TP53 mutant and wild type tumors. The top two panels show the frequency of gains, indicated by the green bars ranging from 0 to $I$, and losses, indicated by the red bars ranging from 0 to -1 , in 62 sporadic breast tumors for each clone. The bottom panel displays the magnitude of the t-statistic for each clone computed based on the smoothed data as described in the Methods. The horizontal dotted lines indicate the statistic cut-off corresponding to the FDR-adjusted $\mathrm{p}$-value of 0.01 (red), 0.05 (blue) and 0.1 (green). Thus, copy number alterations occurring more frequently in TP53 mutant tumors included losses of regions on 3p, 4q, 5q, I5q, I7q and gain of a small region on distal $8 \mathrm{q}$.

whole chromosomes, low level gains and losses affecting extended portions of chromosomes, and amplifications defined as focal regions of increased copy number [14]. Specifically, a clone was declared amplified if it belonged to a copy number segment $<20 \mathrm{Mb}$ and the increase in ratio exceeded the criterion described in the Statitical Methods. The distinction between gains and amplifications can be seen in the copy number profiles in Figure $5 \mathrm{~A}$. A copy number gain spanning $8 \mathrm{q}$ can be seen in the top left profile, while the wide variety in amplicon profiles is evident by comparison of all the profiles. We enumerate low level changes by counting "copy number transitions," the number of changes in the CGH profile from one copy number level to another that occur within chromosomes (see Supplementary Methods for further discussion of aberration finding, Additional file 2). Since the spacing between clones is $\sim 1.5 \mathrm{Mb}$, focal aberrations that fall between clones on the array will be missed. On the other hand, all copy number transitions will be recorded, but the precision with which they will be located on the genome will depend on clone spacing. We note that these copy number analyses found that the number of copy number transitions associated with amplifications varied over a wide range in tumors of all subtypes in both datasets, however the greatest number of amplifications did not occur in the samples with either the smallest or largest number of copy number transitions (Figure 5B).

We tested for associations between gene expression and copy number aberrations by developing a list of 426 genes assigned to functional categories, "DNA replication," "DNA damage/repair," "cell cycle," "mitosis," "centrosome" (centrosome and centrosome cycle) and "telomere" using Gene Ontology Annotation (GOA) terms and reference to the literature (Supplementary Methods and Supplementary Table 3, Additional files 2 and 4). Many of the genes were assigned to more than one process. Expression of 350 of the 426 stability genes could be analyzed in the breast tumor data set. Controlling for estrogen receptor status as a possible confounder, we observed that the telomere functional class was significantly associated with the presence of at least one amplicon, with half of the genes showing positive and half showing negative association (Table 3 ). On the other hand, we found that mitosis, cell cycle, DNA replication and DNA damage/repair functional classes were highly significantly enriched for association with copy number transitions (Table 3). Moreover these associations held

Table 2: TP53 mutations in breast tumors

\begin{tabular}{|c|c|c|c|c|c|c|c|}
\hline $\begin{array}{l}\text { Tumor } \\
\text { Sample }\end{array}$ & CGH Subtype & $\begin{array}{c}\text { TP53 } \\
\text { Mutation }\end{array}$ & $\begin{array}{c}\text { Copy } \\
\text { Number }\end{array}$ & $\begin{array}{l}\text { Loss of } \\
\text { Function' }\end{array}$ & $\begin{array}{c}\text { Gain of } \\
\text { Function }\end{array}$ & Dom. Neg.' & $\mathbf{T S}^{\prime}$ \\
\hline SOOI3 & Complex & Y205D & Loss & NA & NA & NA & NA \\
\hline SOI84 & Complex & $\mathrm{RI} 75 \mathrm{H}$ & Loss & Yes & Yes & Yes & No \\
\hline S0269 & Complex & M237I & Loss & NA & NA & No & NA \\
\hline SI5II & Complex & C275Y & Loss & NA & NA & No & NA \\
\hline SOOOI & Mixed amplifier & Y234C & No Loss & Yes & NA & NA & Yes \\
\hline SO043 & Mixed amplifier & YI63C & No Loss & Yes & Yes & No & NA \\
\hline S0055 & Mixed amplifier & 196 Stop & Loss & NA & NA & No & NA \\
\hline SOI 26 & Mixed amplifier & intron 5/6 & Loss & NA & NA & NA & NA \\
\hline SI503 & Mixed amplifier & CI76Y & Loss & Yes & NA & Yes & NA \\
\hline SI579 & Mixed amplifier & G245C & Loss & Yes & NA & Yes & NA \\
\hline
\end{tabular}

'From IARC TP53 database release, RI0, July 2005 [48]. Function assayed in either human or yeast. Dom. Neg., Dominant Negative. TS,

Temperature sensitive. 


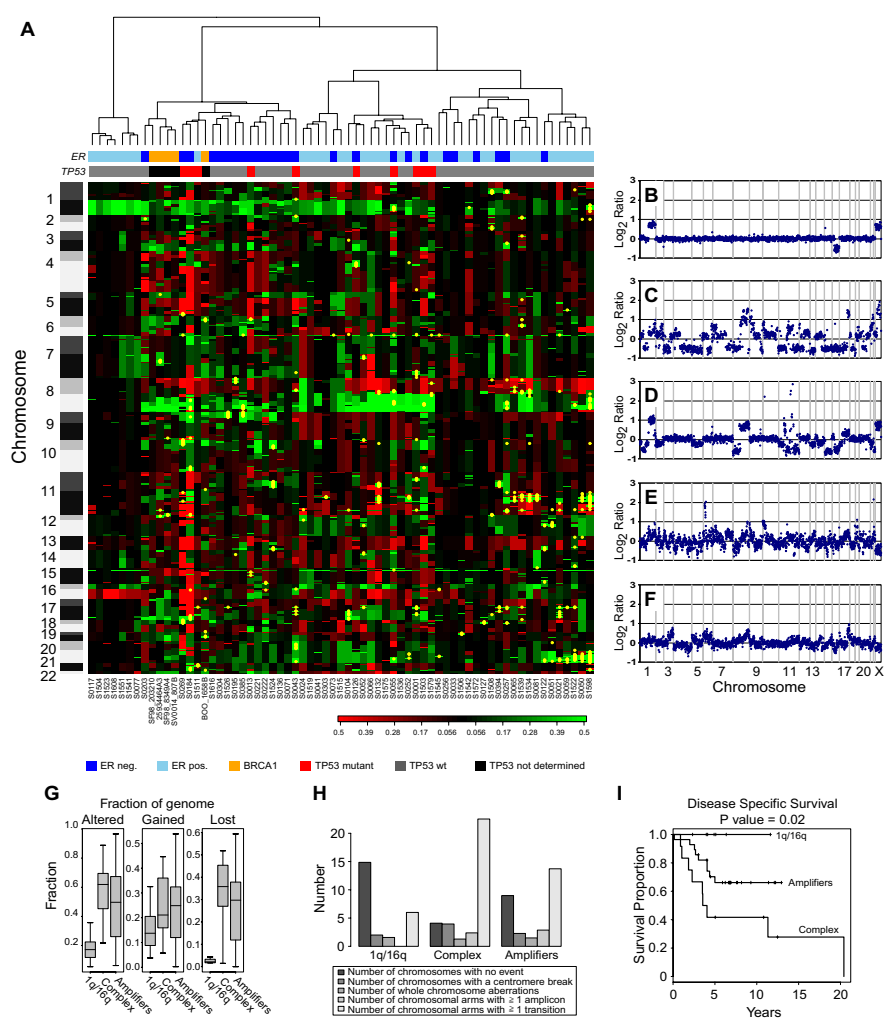

Figure 3

Genomic analysis of breast tumors reveals three subtypes. A. Hierarchical clustering of 62 ductal invasive breast tumors and five BRCAI mutant tumors based on their genome-wide DNA copy number profiles. Individual clones are represented as rows, ordered by chromosome and genome position according to the July 2003 freeze of the human genome. Clones on the p-arm and q-arm of chromosomes are indicated in shades of dark gray (odd numbered chromosomes) or light gray (even numbered chromosomes). Acrocentric chromosomes are shown in dark or light gray. Columns represent individual tumor samples. The estrogen receptor status of the tumors is shown in shades of blue (dark blue $=E R$ negative, light blue $=E R$ positive), BRCAI mutant tumors are indicated in orange, and TP53 mutation status is indicated with a maroon box for TP53 mutant tumors, a gray box for tumors with no detected mutation and a black box if the TP53 status is unknown. Copy number losses are indicated in red, gains in green and amplifications as yellow dots. Three main clusters are evident. B-F. Genomewide copy number aberrations profiles of sporadic and hereditary (BRCAI) breast tumors are plotted as the normalized $\log _{2}$ ratio for each clone sorted by chromosome and ordered according to genome position from the p-arm to the q-arm. Normalized copy number ratios of genomic DNA are shown for a tumor from the Iq/I6q cluster with few copy number changes including gain of $\mathrm{Iq}$ and loss of $\mathrm{I} 6 \mathrm{q}(\mathrm{B})$, a tumor from the ER negative, complex cluster showing many low level chromosome changes and few amplifications (C), a tumor from the amplifier cluster with low level gains and losses and amplifications (D) and tumors from patients with mutations in BRCAI (E and F). G. Numbers and types of copy number aberrations in breast tumor subtypes. The mean numbers of whole chromosome copy number changes, copy number transitions and amplifications were determined for the tumors within each subtype. $\mathbf{H}$. Numbers and types of copy number aberrations in breast tumor subtypes. The mean numbers of chromosomes showing no copy number change, whole chromosome copy number changes, copy number transitions, copy number transitions at centromeres and amplifications were determined for the tumors within each subtype. I. Association with disease-specific survival. Significance of the log-rank test was used to assess the association between a genomic subclass and survival phenotypes. The significance was declared at $p<0.05$. Patients with complex tumors experienced significantly worse outcome compared to the other groups. 


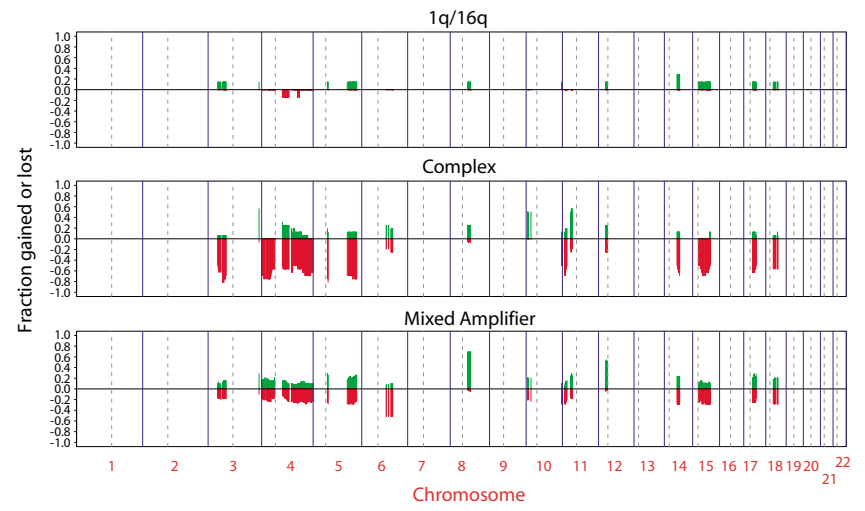

Figure 4

Copy number changes more frequently associated with one subtype. Frequency for each clone of gains and losses, which were uniquely present in more than $50 \%$ of samples of one subtype and in less than $30 \%$ of samples in other subtypes. Gains are indicated by green bars, ranging from 0 to I, and losses, by the red bars ranging from 0 to $-\mathrm{I}$ for each clone.

when only tumors of the complex subtype were considered, indicating that the results are not due to confounding between the large number of low level transitions and complex subtype. Expression of 146 stability genes was significantly associated with the number of copy number transitions (false discovery rate, FDR $<0.05$ ). Most associations were positive (120/146 genes), indicating that enhanced expression of these genes was associated with greater numbers of copy number transitions (Figure 7). The number of amplifications was associated with mitosis, cell cycle and to a lesser extent, DNA replication categories. Again, this relation held when considering all samples, as well as only samples within the mixed amplifier subtype. Twenty-five individual genes were associated with number of amplifications (FDR $<0.05)$ and 21 were in common with the group of genes associated with copy number transitions (Figure 7). Here too, we observed that most associations were positive (21/25 genes), indicating that increased expression of the genes was observed in tumors with more amplifications. Finally, we investigated how the GOA categories represented by our list of 426 stability genes ranked among all known GOA categories with respect to associations with frequency of copy number aberrations by considering all probes measured by the expression analysis. In this subsequent unsupervised analysis, we found enrichment for genes associated with copy number transitions (FDR $<0.05)$ in the same functional classes, e.g. mitosis, cell cycle, cell division and DNA replication (Holm adjusted p-value $<0.005$ ), providing further support for these associations.
We noted that the 146 stability genes associated with numbers of copy number transitions included E2F1, and they are significantly enriched for genes known to be targets of E2F1 ( $<<2 \times 10-6$, Fisher exact test, Figure 7). Moreover the expression levels of known E2F1 target genes were highly correlated with E2F1 expression ( $\mathrm{p}<2$ $\times$ 10-10, Supplementary Table 4, Additional file 5). These observations provide in vivo validation of the in vitro determinations of E2F1 target genes. They are also consistent with deregulation of E2F being a major contributor to genomic instability affecting numbers of copy number transitions and amplifications. Taken together these observations suggest that telomere attrition and deregulated expression of genes in the other functional classes, particularly those that are targets of E2F, contribute to the numbers of chromosomal alterations.

\section{Discussion}

Our analysis of large numbers of breast tumors by array CGH revealed variety in the numbers and types of copy number alterations in the tumor genomes. In the ductal invasive breast tumors reported here, three subtypes were distinguished by copy number alterations. The subtypes differed with respect to the numbers and types of aberrations, as well as patient survival. The $1 \mathrm{q} / 16 \mathrm{q}$ subtype with very few copy number alterations in addition to gain of $1 \mathrm{q}$ and loss of $16 \mathrm{q}$ was associated with the best patient outcome, consistent with other studies. Searches for tumor suppressor gene(s) on $16 \mathrm{q}$ have failed to find mutations in candidate genes in the region in ductal invasive breast cancer, although mutations in E cadherin and loss of $16 \mathrm{q}$ are characteristic of lobular breast tumors. Two genes involved in telomere maintenance, TERF2 and TERF2IP were among those ruled out as tumor suppressors on $16 \mathrm{q}$, as was E2F4 [15-17]. The stability of the genome of these tumors also suggests that copy number alterations of these and other stability genes mapping within the aberrant regions, $+1 \mathrm{q}$ and $-16 \mathrm{q}$ are less likely to contribute to chromosomal level instability in breast cancer.

Complex tumors with extensive chromosomal level instability were associated with poor patient survival. They are similar to $B R C A 1$ hereditary tumors in their copy number alterations [18,19] (Figure 3). BRCA1 participates in a number of cell functions that maintain genome integrity either directly through double strand break repair or indirectly through maintenance of checkpoints at G1, S and mitosis [20-22]. Thus, it is possible that BRCA1 $[23,24]$ or the genes/pathways that interact with $B R C A 1$ are defective in this subtype either through mutation, silencing or copy number mediated dosage effects. We note that the copy number loss on $17 \mathrm{q}$ associated with this subtype includes the BRCA1 locus (9/16 tumors, Figure 4$)$. 
A
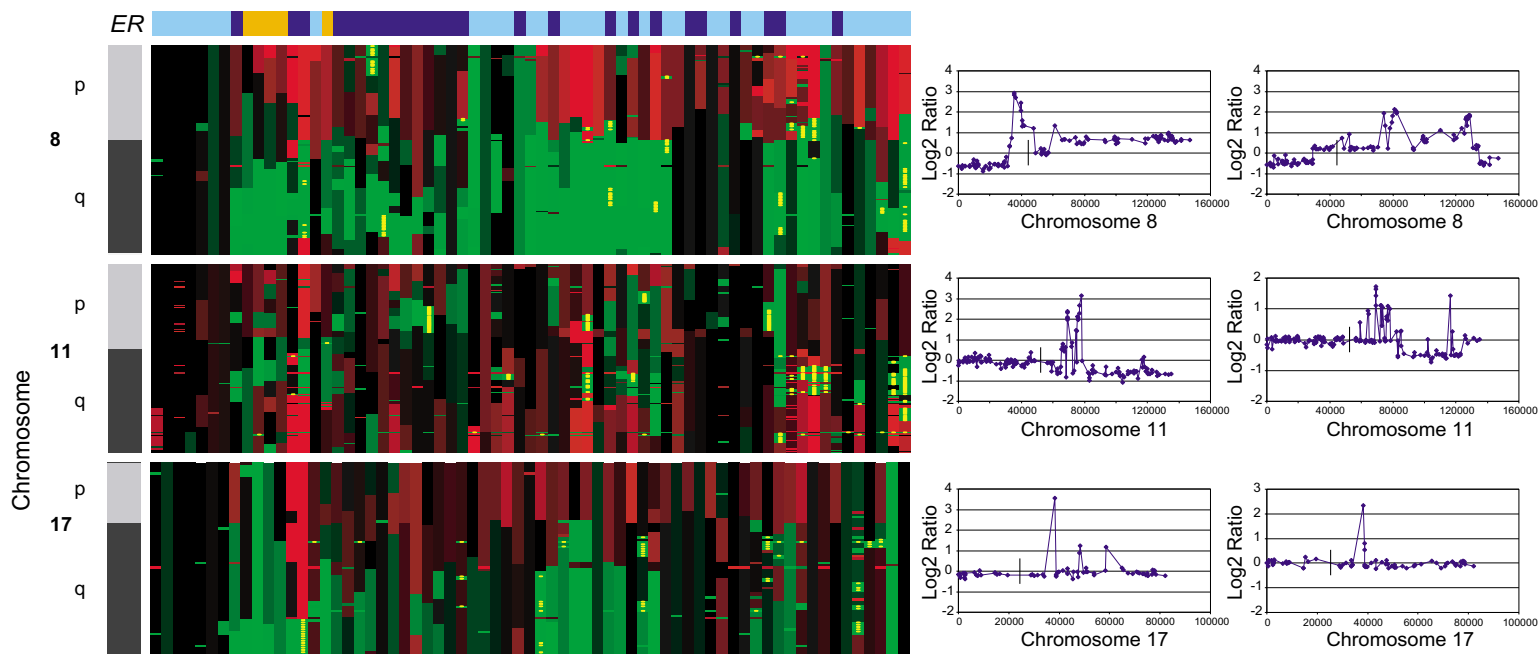

$2^{p}$
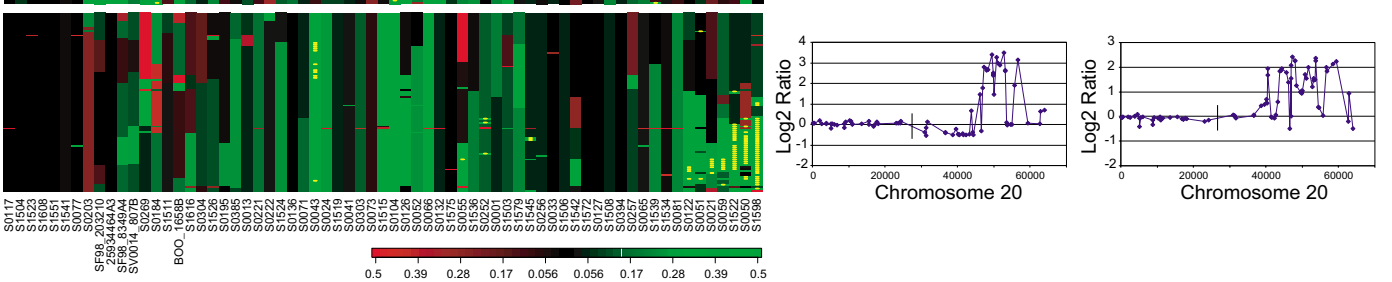

ER neg. ER pos. BRCA1

B

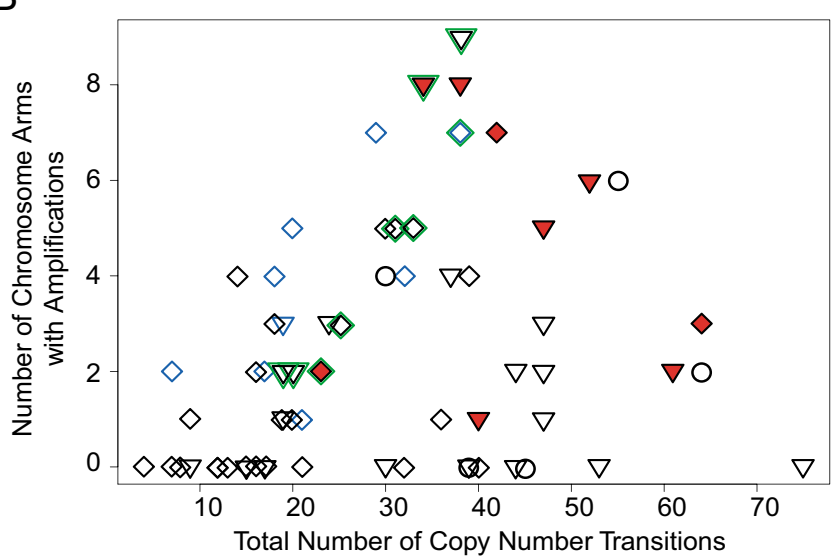

$\begin{array}{llllll}\nabla & \text { ER negative } & \diamond \nabla & \text { ERBB2 amplified } & \diamond & \text { TP53 mutant } \\ \diamond & \text { ER positive } & \diamond \nabla & \text { CCND1 amplified } & \bigcirc & \text { BRCA1 mutant }\end{array}$

Figure 5

Overview of most frequent DNA amplifications on chromosomes 8, 11,17 and 20 in 62 breast tumors determined by genome-wide array CGH. A. Heat map depiction of aberrations on chromosomes $8,11,17$ and 20 and typical chromosome copy number profiles showing amplifications of $8 p$ (including FGFRI), I IqI3 (including CCNDI), I7q (ERBB2) and regions on 20q (including ZNF2 I 7). Note that the chromosome II copy number profiles vary depending on whether amplification of chromosome 8 is also present. In both cases copy number losses distal to the amplicon are observed, however in the absence of chromosome 8 amplification (right), the region of loss extends distally from the amplified region, whereas, when chromosome 8 was amplified (left), the copy number loss includes regions proximal as well as distal to CCNDI. B. For each tumor, the numbers of copy number transitions is compared to the number of chromosome arms with at least one amplification. 


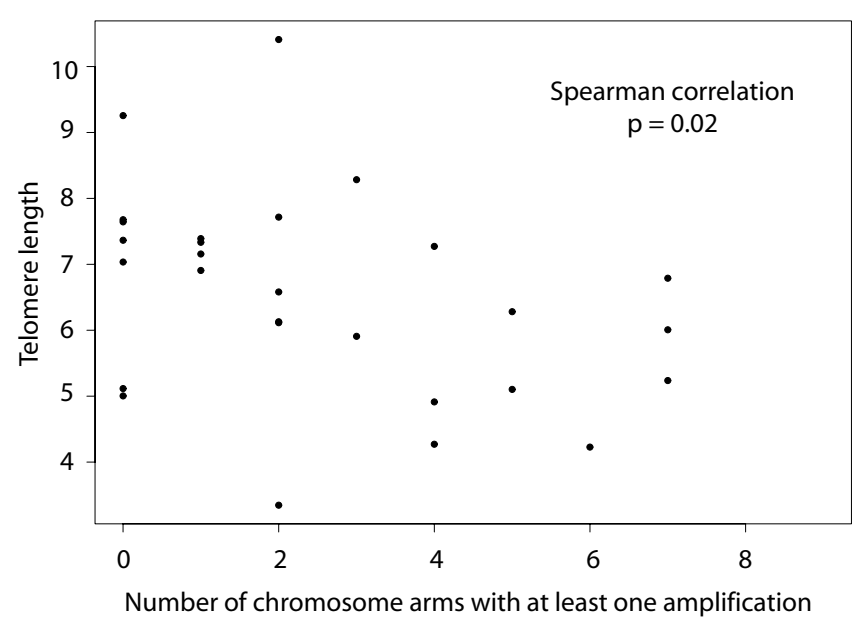

Figure 6

Telomere length measurements in $\mathbf{2 8}$ breast tumor samples. Plotted is the telomere length determined by Southern blotting relative to the number of chromosome arms with amplification. A significant inverse correlation is evident.

The discrimination of breast tumor subtypes based on copy number aberrations led us to investigate possible associations of copy number aberration types with alterations in processes/genes involved in maintenance of genome stability. We observed shorter telomeres in tumors with greater numbers of amplifications, consistent with telomere attrition promoting this type of copy number aberration in breast tumors. Telomere dysfunction, often referred to as "telomere crisis" has been implicated in amplification, particularly by breakage-fusionbridge processes. On the other hand, our analyses of stability gene expression in relation to copy number aberration types found that expression of genes in the functional classes; "mitosis," "cell cycle," "replication," and "DNA damage/repair" were associated with greater numbers of copy number transitions. Furthermore, a subsequent analysis found significant enrichment for these same classes among all GOA groups when analyzed with GOStats [25]. The number of amplicons was associated with similar functional groups, "mitosis" and "cell cycle." Many of these genes are E2F targets [26-36] and therefore potentially coordinately deregulated due to $\mathrm{Rb}$ pathway defects [37]. Abrogation of Rb pathway function is frequent in breast tumors by loss of expression of $\mathrm{Rb}$ or altered expression of inhibitors of Rb activity (e.g. loss/ silencing of CDKN2A (p16) and amplification and/or over expression of CCND1,CDK4, CDK6) (Figure 7). It is interesting to note that whereas E2F1 is up-regulated in breast tumors, its expression is low in prostate tumors [38], which typically have genomes with fewer copy number changes than most ductal invasive breast cancers [39]. For example, in an array CGH dataset of 64 primary prostate tumor samples [39], the median number of copy number transitions was 13 per tumor genome compared to 30 in our primary breast tumor samples $\left(\mathrm{p}<5 \times 10^{-9}\right.$, Wilcoxon rank sum test). Mechanistic support for a central role of E2F1 in genomic instability comes from a recent report that elevated numbers of DNA double strand breaks are present in cell lines with deregulated E2F1 and $\mathrm{Rb}$ deficiency [40].

Chromosomal instability has been observed in vitro when many of these E2F target genes (Figure 7) associated with replication, DNA repair, cell cycle control and the mitotic checkpoint are mutated, knocked out or knocked down using siRNA $[8,41,42]$. Contrary to expectation, we observed that greater chromosomal instability in breast tumors is associated with increased expression levels of many of these genes, even though they have loss of function instability phenotypes. These assays further demonstrate that loss of a single copy of some of the genes results instability or cancer prone phenotypes. Genes that have been shown to be haploinsufficient in this way and that are among those we identified as showing significant association with the number of copy number aberrations in our tumors (FDR < 0.05) include RAD17, ATM and $R B 1$, which are expressed at lower levels in tumors with more copy number changes. These genes are also negatively correlated with E2F1 expression. Other genes showing haploinsufficiency in vitro, MAD2L1, PLK4, BUB1B and CHEK1 show enhanced expression in association with number of chromosomal changes and are positively correlated with E2F1 expression (Supplementary Table 4, Additional file 5). As all seven of the above mentioned genes with haploinsufficiency phenotypes map to regions of frequent loss in breast tumors and genetic instability phenotypes are associated with deficiency in these genes, we asked whether loss of function might play a role in the subset of tumors in which there is a copy number loss of the locus. Specifically, we asked if their expression levels were down regulated when there is a copy number loss. Although 118 of the genome stability genes showed highly significant reduction in expression in tumors in which the locus was lost (FDR $<0.05$, one-sided Wilcoxon rank sum test), we found little difference in expression level with copy number loss for MAD2L1, PLK4, ATM and $R B 1$, whereas $B U B 1 B$ was increased in expression in tumors with loss of the locus (Supplementary Table 4, Additional file 5). Only expression of RAD17 was significantly reduced when lost (unadjusted $\mathrm{p}=8 \times 10^{-4}$, Wilcoxon rank sum test), suggesting that RAD17 might be haploinsufficient in tumors with copy number loss of the locus at $5 \mathrm{q} 13$.

Our observations in tumors support the hypothesis that global alteration of expression of genes involved in processes such as chromosome segregation and maintenance 
Table 3: Association of expression of functional classes with copy number aberration types

\begin{tabular}{cccc}
\hline Class & Copy Number Transitions & Amplifications & At least one Amplicon \\
Mitosis & 0 & $4.6 \times 10^{-4}$ & 0.15 \\
Cell Cycle & 0 & $5.2 \times 10^{-5}$ & 0.02 \\
DNA Replication & $3.1 \times 10^{-12}$ & 0.04 & 0.25 \\
Centrosome & 0.28 & 0.52 & 0.25 \\
Telomere & 0.02 & 0.06 & $2.9 \times 10^{-4}$ \\
DNA Damage/Repair & $7.3 \times 10^{-9}$ & 0.06 & 0.2 \\
\hline
\end{tabular}

of genome integrity, driven by deregulation of E2F, underlies much of the chromosomal instability in breast tumors. Furthermore gene expression appears to be relatively up-regulated. On the one hand, this observation seems contradictory in light of the phenotypes resulting from mutational analyses of genes involved in maintenance of genome stability. Such in vitro studies have generally assessed the consequences of functional deficiency one gene at a time and have found that individually many genes have loss of function instability phenotypes. On the other hand, as many of these genes participate in multiprotein complexes that depend on proper stoichiometry for function, alterations resulting in overproduction or deficiency are likely to have similar or related phenotypes (reviewed in [43]). Indeed, in mammalian cells, instability phenotypes have been reported in association with both up and down regulation of genes such as MAD2L1 [8,41], ATR [44,45], PLK4 [46] and AURKA [47]. Further studies will be required not only to assess instability phenotypes when expression levels are increased, but also how phenotypes might vary when multiple genes are upregulated.

In tumors, changes in gene dosage due to low level copy number alterations may also lead to small alterations in expression of multiple genes, which together could contribute to dysfunction of processes manipulating the genome, resulting in more error prone cell division cycles. Thus, during tumor progression, genome instability may be enhanced not only by deregulation of E2F, but also by the acquisition of greater numbers of copy number changes encompassing more genes involved in genome maintenance. Since genetic instability is an on-going feature of tumors, allowing them to evolve resistance to therapy, the ability to recognize the active mechanisms of instability in tumors may help to guide therapeutic decisions.

\section{Conclusion}

Application of array CGH to the study of breast tumors found three subtypes. Investigation of the numbers and types of copy number alterations in tumors and their association with differential expression of genes likely to play a role in manipulation or maintenance of the genome implicated telomere dysfunction in the propensity to amplify. On the other hand, the number of lower magnitude gains and losses of chromosomal segments was associated with differential expression of genes which were significantly enriched for the known targets of E2F, supporting the hypothesis that deregulation of E2F underlies much of the chromosomal instability in breast tumors. Furthermore, we observed enhanced expression of most E2F target genes, indicating that over expression rather than deficiency was associated with genetic instability. These observations provide a possible explanation for the failure to find mutations in sporadic tumors in genes that have roles in maintenance or manipulation of the genome.

\section{Competing interests}

The author(s) declare that they have no competing interests.

\section{Authors' contributions}

JF performed the statistical analysis and contributed to writing the manuscript, AMS and BY performed the array CGH and contributed to writing the manuscript, HL performed the telomere length measurements, AO performed statistical analysis, RS performed array CGH, SD directed the dissection of tumor tissue and extraction of nucleic acids, TT contributed to the development of analytical procedures for image analysis and performed the image analysis, BML was responsible for pathologic diagnosis, AJN contributed to the development of analytical procedures, JM and JZ manage and direct the UCSF Comprehensive Cancer Center Risk Program and provided access to BRCA1 mutant tumors, KC and SD performed the array CGH for breast study II, HF was responsible for the expression array measurements, JG and FW directed breast study II and shared their unpublished data, DP participated in technology development that made this study possible, DGA directed the study, participated in technology development and was responsible for preparation of the manuscript. 


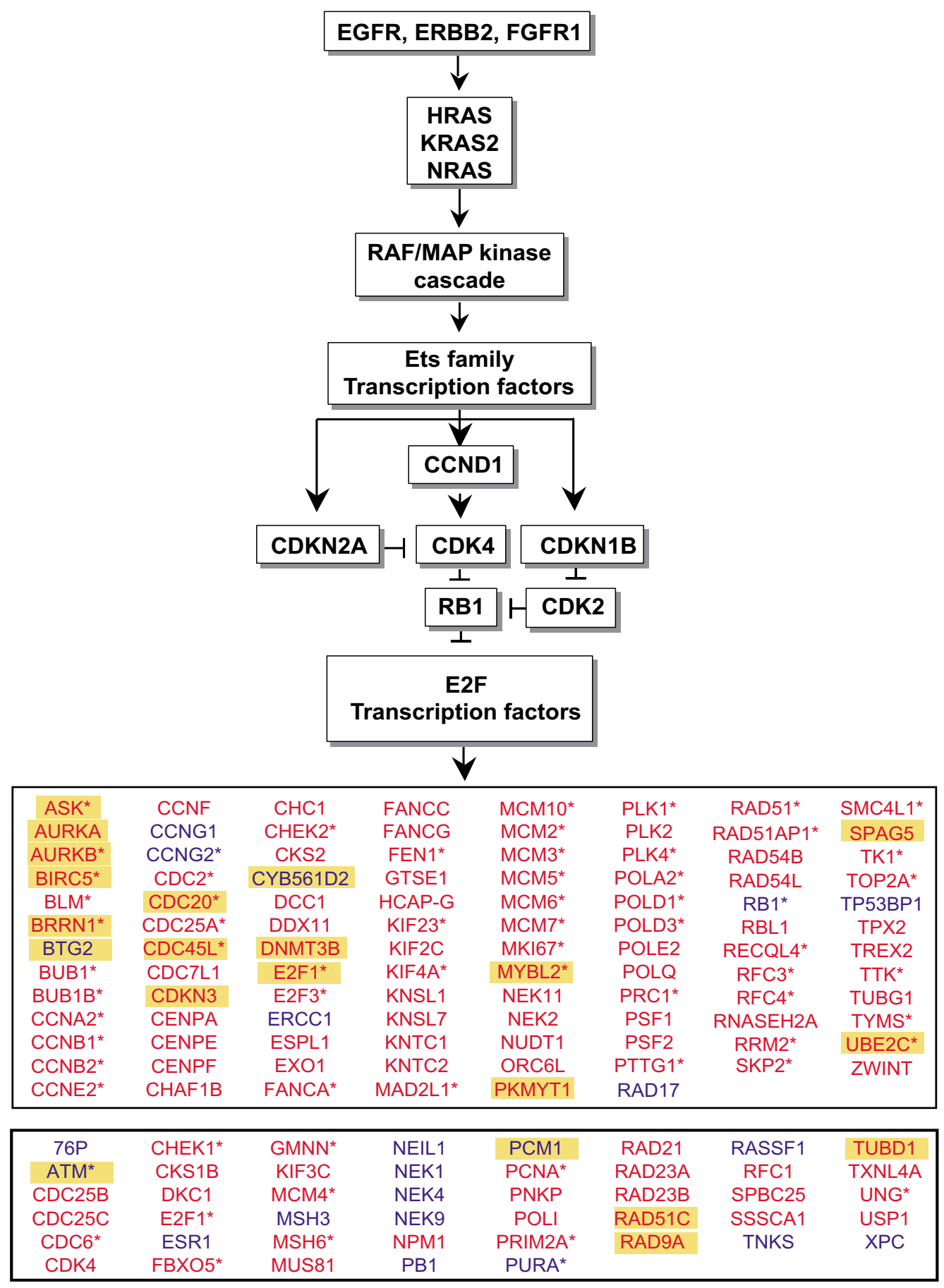

\section{Figure 7}

Signaling pathway and E2F responsive genes associated with numbers of copy number transitions and amplifications. The receptor signaling cascade impinging on Rb includes a number of up-stream genes that are frequently altered in cancers and result in deregulation of the E2F family of transcription factors through inhibition or loss of Rb function. Here, we show genes enriched for association with numbers of copy number transitions (FDR < 0.05 ). Genes, which have been identified as E2F targets (asterisk) and/or are correlated with E2FI expression levels (|Pearson correlation| $>0.3$ ) are shown in the top box. Genes which showed less correlation with E2FI are shown in the bottom box. Genes also associated with numbers of amplifications (FDR < 0.05) are highlighted in yellow. Four additional genes associated with amplifications (FDR < 0.05$)$ are ASCIZ, MSH5, RAEI and TDG. Red, increased expression. Blue, decreased expression. 


\section{Additional material}

\section{Additional File 1}

Additional file 1 - Supplementary Table 1. Patient CharacteristicsProvides information on patient samples.

Click here for file

[http://www.biomedcentral.com/content/supplementary/14712407-6-96-S1.xls]

\section{Additional File 2}

Additional file 2 - Supplementary Statistical Methods Provides a detailed description of statistical methods.

Click here for file

[http://www.biomedcentral.com/content/supplementary/14712407-6-96-S2.doc]

\section{Additional File 3}

Additional file 3 - Supplementary Table 2. Array CGH data Provides array $\mathrm{CGH} \log _{2}$ ratios for clones and genome order for all samples.

Click here for file

[http://www.biomedcentral.com/content/supplementary/14712407-6-96-S3.xls]

\section{Additional File 4}

Additional file 4 - Supplementary Table 3. Genome stability genes and associations with copy number aberrations Provides information on 426 genome stability genes and associations with copy number aberration types.

Click here for file

[http://www.biomedcentral.com/content/supplementary/14712407-6-96-S4.xls]

\section{Additional File 5}

Additional file 5 - Supplementary Table 4. Genome stability genes and their relation to $\mathrm{E} 2 \mathrm{~F}$. Table provides information on whether the genes are known targets of $\mathrm{E} 2 \mathrm{~F} 1$, correlation of their expression with E2F1, correlation of their expression with copy number in samples in which copy number of the locus is reduced.

Click here for file

[http://www.biomedcentral.com/content/supplementary/14712407-6-96-S5.xls]

\section{Acknowledgements}

We thank members of the UCSF Comprehensive Cancer Center Genome Analysis Shared Resource Facility, Sonia Mirza, Julie Weng, Maimie Yu, and Facility Manager, David Ginzinger for carrying out the TP53 sequencing. This work was supported by NIH grants CA9042 I and CA I 01359 (JF, AMS, BY, HL, DGA), CA789I3 (RS, TT, ANJ, DP), and CA58207, the Office of Health and Environmental Research of the U.S. Department of Energy (Contract DE-AC03-76SF00098) and the Avon foundation (SD, BML, JM, JZ, KC, SD, HF, JWG, FW).

\section{References}

I. Albertson DG, Collins C, McCormick F, Gray JW: Chromosome aberrations in solid tumors. Nat Genet 2003, 34:369-376.

2. Cahill DP, Lengauer C, Yu J, Riggins GJ, Willson JK, Markowitz SD, Kinzler KW, Vogelstein B: Mutations of mitotic checkpoint genes in human cancers. Nature 1998, 392:300-303.

3. Wang Z, Cummins JM, Shen D, Cahill DP, Jallepalli PV, Wang TL, Parsons DW, Traverso G, Awad M, Silliman N, Ptak J, Szabo S, Willson
JK, Markowitz SD, Goldberg ML, Karess R, Kinzler KW, Vogelstein B, Velculescu VE, Lengauer C: Three classes of genes mutated in colorectal cancers with chromosomal instability. Cancer Res 2004, 64:2998-300I.

4. Bartkova J, Horejsi Z, Koed K, Kramer A, Tort F, Zieger K, Guldberg P, Sehested M, Nesland JM, Lukas C, Orntoft T, Lukas J, Bartek J: DNA damage response as a candidate anti-cancer barrier in early human tumorigenesis. Nature 2005, 434:864-870.

5. Gorgoulis VG, Vassiliou LV, Karakaidos P, Zacharatos P, Kotsinas A Liloglou T, Venere M, Ditullio RA Jr, Kastrinakis NG, Levy B, Kletsas D, Yoneta A, Herlyn M, Kittas C, Halazonetis TD: Activation of the DNA damage checkpoint and genomic instability in human precancerous lesions. Nature 2005, 434:907-913.

6. Feldser DM, Hackett JA, Greider CW: Telomere dysfunction and the initiation of genome instability. Nat Rev Cancer 2003, 3:623-627.

7. Mao JH, Perez-Losada J, Wu D, Delrosario R, Tsunematsu $R$, Nakayama KI, Brown K, Bryson S, Balmain A: Fbxw7/Cdc4 is a p53. dependent, haploinsufficient tumour suppressor gene. Nature 2004, 432:775-779.

8. Hernando E, Nahle Z, Juan G, Diaz-Rodriguez E, Alaminos M, Hemann M, Michel L, Mittal V, Gerald W, Benezra R, Lowe SW, Cordon-Cardo C: $\mathbf{R b}$ inactivation promotes genomic instability by uncoupling cell cycle progression from mitotic control. Nature 2004, 430:797-802.

9. Albertson DG, Ylstra B, Segraves R, Collins C, Dairkee SH, Kowbe D, Kuo WL, Gray JW, Pinkel D: Quantitative mapping of amplicon structure by array CGH identifies CYP24 as a candidate oncogene. Nat Genet 2000, 25: |44- I 46.

10. Pinkel D, Segraves R, Sudar D, Clark S, Poole I, Kowbel D, Collins C, Kuo WL, Chen C, Zhai Y, Dairkee SH, Ljung BM, Gray JW, Albertson DG: High resolution analysis of DNA copy number variation using comparative genomic hybridization to microarrays. Nat Genet 1998, 20:207-2II.

II. Snijders AM, Schmidt BL, Fridlyand J, Dekker N, Pinkel D, Jordan RC, Albertson DG: Rare amplicons implicate frequent deregulation of cell fate specification pathways in oral squamous cell carcinoma. Oncogene 2005, 24:4232-4242.

12. Snijders AM, Nowak N, Segraves R, Blackwood S, Brown N, Conroy J, Hamilton G, Hindle AK, Huey B, Kimura K, Law S, Myambo K, Palmer J, Ylstra B, Yue JP, Gray JW, Jain AN, Pinkel D, Albertson DG: Assembly of microarrays for genome-wide measurement of DNA copy number. Nat Genet 200I, 29:263-264.

13. Loo LW, Grove DI, Williams EM, Neal CL, Cousens LA, Schubert EL, Holcomb IN, Massa HF, Glogovac J, Li Cl, Malone KE, Daling JR, Delrow JJ, Trask BJ, Hsu L, Porter PL: Array comparative genomic hybridization analysis of genomic alterations in breast cancer subtypes. Cancer Res 2004, 64:854I-8549.

14. Snijders AN, Fridlyand J, Mans D, Segraves R, Jain AN, Pinkel D, Albertson DG: Shaping tumor and drug resistant genomes by instability and selection. Oncogene 2003, 22:4370-4379.

15. Rakha EA, Armour JA, Pinder SE, Paish CE, Ellis IO: High-resolution analysis of 16q22. I in breast carcinoma using DNA amplifiable probes (multiplex amplifiable probe hybridization technique) and immunohistochemistry. Int J Cancer 2005, I | 4:720-729.

16. Rakha EA, Pinder SE, Paish EC, Robertson JF, Ellis IO: Expression of E2F-4 in invasive breast carcinomas is associated with poor prognosis. J Pathol 2004, 203:754-76I.

17. van Wezel T, Lombaerts M, van Roon EH, Philippo K, Baelde HJ, Szuhai K, Cornelisse C], Cleton-Jansen AM: Expression analysis of candidate breast tumour suppressor genes on chromosome I6q. Breast Cancer Res 2005, 7:R998-RI004.

18. Jonsson G, Naylor TL, Vallon-Christersson J, Staaf J, Huang J, Ward MR, Greshock JD, Luts L, Olsson H, Rahman N, Stratton M, Ringner $M$, Borg A, Weber BL: Distinct genomic profiles in hereditary breast tumors identified by array-based comparative genomic hybridization. Cancer Res 2005, 65:76I2-762I.

19. Wessels LF, van Welsem T, Hart AA, van't Veer LJ, Reinders MJ, Nederlof PM: Molecular classification of breast carcinomas by comparative genomic hybridization: a specific somatic genetic profile for BRCAI tumors. Cancer Res 2002, 62:7110-7117.

20. Scully R, Xie A, Nagaraju G: Molecular functions of BRCAI in the DNA damage response. Cancer Biol Ther 2004, 3:52 I-527. 
21. Wang $\mathrm{RH}, \mathrm{Yu} \mathrm{H}$, Deng CX: A requirement for breast-cancerassociated gene I (BRCAI) in the spindle checkpoint. Proc Natl Acad Sci U S A 2004, I 0 I: I7 I08-I7II3.

22. Yoshida K, Miki Y: Role of BRCAI and BRCA2 as regulators of DNA repair, transcription, and cell cycle in response to DNA damage. Cancer Sci 2004, 95:866-87I.

23. Staff S, Isola J, Tanner M: Haplo-insufficiency of BRCAI in sporadic breast cancer. Cancer Res 2003, 63:4978-4983.

24. Wei M, Grushko TA, Dignam J, Hagos F, Nanda R, Sveen L, Xu J, Fackenthal J, Tretiakova M, Das S, Olopade OI: BRCAI promoter methylation in sporadic breast cancer is associated with reduced BRCAI copy number and chromosome 17 aneusomy. Cancer Res 2005, 65:10692-10699.

25. Beissbarth T, Speed TP: GOstat: find statistically overrepresented Gene Ontologies within a group of genes. Bioinformatics 2004, 20: | 464- | 465

26. Berkovich E, Ginsberg D: ATM is a target for positive regulation by E2F-I. Oncogene 2003, 22:161-I67.

27. Carrassa L, Broggini M, Vikhanskaya F, Damia G: Characterization of the 5'flanking region of the human Chkl gene: identification of E2F I functional sites. Cell Cycle 2003, 2:604-609.

28. Ishida S, Huang E, Zuzan H, Spang R, Leone G, West M, Nevins JR: Role for E2F in control of both DNA replication and mitotic functions as revealed from DNA microarray analysis. Mol Cell Biol 200I, 2 I:4684-4699.

29. Iwanaga R, Komori H, Ishida S, Okamura N, Nakayama K, Nakayama $\mathrm{KI}$, Ohtani K: Identification of novel E2F I target genes regulated in cell cycle-dependent and independent manners. Oncogene 2005.

30. Jiang Y, Saavedra HI, Holloway MP, Leone G, Altura RA: Aberrant regulation of survivin by the RB/E2F family of proteins. J Biol Chem 2004, 279:405 I I-40520.

31. Kel AE, Kel-Margoulis OV, Farnham PJ, Bartley SM, Wingender E, Zhang MQ: Computer-assisted identification of cell cyclerelated genes: new targets for E2F transcription factors. Mol Biol 2001, 309:99-120.

32. Muller H, Bracken AP, Vernell R, Moroni MC, Christians F, Grassilli E, Prosperini E, Vigo E, Oliner JD, Helin K: E2Fs regulate the expression of genes involved in differentiation, development, proliferation, and apoptosis. Genes Dev 200I, I 5:267-285.

33. Polager S, Kalma Y, Berkovich E, Ginsberg D: E2Fs up-regulate expression of genes involved in DNA replication, DNA repair and mitosis. Oncogene 2002, 21 :437-446.

34. Ren B, Cam H, Takahashi Y, Volkert T, Terragni J, Young RA, Dynlacht BD: E2F integrates cell cycle progression with DNA repair, replication, and $\mathbf{G ( 2 ) / M}$ checkpoints. Genes Dev 2002, 1 6:245-256.

35. Vernell $\mathrm{R}$, Helin $\mathrm{K}$, Muller $\mathrm{H}$ : Identification of target genes of the pI6INK4A-pRB-E2F pathway. I Biol Chem 2003, 278:46 I 24-46 I 37.

36. Markey $M$, Siddiqui $H$, Knudsen ES: Geminin is targeted for repression by the retinoblastoma tumor suppressor pathway through intragenic E2F sites. I Biol Chem 2004, 279:29255-29262

37. Black EP, Huang E, Dressman H, Rempel R, Laakso N, Asa SL, Ishida $S$, West M, Nevins JR: Distinct gene expression phenotypes of cells lacking $\mathbf{R b}$ and Rb family members. Cancer Res 2003, 63:3716-3723.

38. Zacharatos $P$, Kotsinas A, Evangelou K, Karakaidos P, Vassiliou LV, Rezaei N, Kyroudi A, Kittas C, Patsouris E, Papavassiliou AG, Gorgoulis VG: Distinct expression patterns of the transcription factor E2F-I in relation to tumour growth parameters in common human carcinomas. J Pathol 2004, 203:744-753.

39. Paris PL, Andaya A, Fridlyand J, Jain AN, Weinberg V, Kowbel D, Brebner JH, Simko J, Watson JE, Volik S, Albertson DG, Pinkel D, Alers JC, van der Kwast TH, Vissers KJ, Schroder FH, Wildhagen MF, Febbo PG, Chinnaiyan AM, Pienta KJ, Carroll PR, Rubin MA, Collins $C$, van Dekken $H$ : Whole genome scanning identifies genotypes associated with recurrence and metastasis in prostate tumors. Hum Mol Genet 2004, I 3:1303-13 I3.

40. Pickering MT, Kowalik TF: Rb inactivation leads to E2F I-mediated DNA double-strand break accumulation. Oncogene 2005

41. Michel LS, Liberal V, Chatterjee A, Kirchwegger R, Pasche B, Gerald W, Dobles M, Sorger PK, Murty VV, Benezra R: MAD2 haplo-insufficiency causes premature anaphase and chromosome instability in mammalian cells. Nature 200I, 409:355-359.
42. Wang X, Zou L, Zheng H, Wei Q, Elledge SJ, Li L: Genomic instability and endoreduplication triggered by RADI 7 deletion. Genes Dev 2003, I 7:965-970.

43. Ouspenski II, Elledge SJ, Brinkley BR: New yeast genes important for chromosome integrity and segregation identified by dosage effects on genome stability. Nucleic Acids Res 1999 27:300I-3008.

44. Cortez D, Guntuku S, Qin J, Elledge SJ: ATR and ATRIP: partners in checkpoint signaling. Science 200I, 294:I7|3-I7/6.

45. Smith L, Liu SJ, Goodrich L, Jacobson D, Degnin C, Bentley N, Carr A, Flaggs G, Keegan K, Hoekstra M, Thayer MJ: Duplication of ATR inhibits MyoD, induces aneuploidy and eliminates radiationinduced GI arrest. Nat Genet 1998, I 9:39-46.

46. Habedanck R, Stierhof YD, Wilkinson CJ, Nigg EA: The Polo kinase Plk4 functions in centriole duplication. Nat Cell Biol 2005, 7:1|40-I|46

47. Bischoff JR, Plowman GD: The Aurora/lpIlp kinase family: regulators of chromosome segregation and cytokinesis. Trends Cell Biol 1999, 9:454-459.

48. Olivier M, Eeles R, Hollstein M, Khan MA, Harris CC, Hainaut P: The IARC TP53 database: new online mutation analysis and recommendations to users. Hum Mutat 2002, |9:607-6|4.

\section{Pre-publication history}

The pre-publication history for this paper can be accessed here:

http://www.biomedcentral.com/1471-2407/6/96/prepub Publish with Biomed Central and every charge

"BioMed Central will be the most significant development for disseminating the results of biomedical research in our lifetime. "

Sir Paul Nurse, Cancer Research UK

Your research papers will be:

- available free of charge to the entire biomedical community

- peer reviewed and published immediately upon acceptance

- cited in PubMed and archived on PubMed Central

- yours - you keep the copyright

BioMedcentral 